\title{
Herramientas didácticas para la enseñanza universitaria de la Traduc- ción e Interpretación online
}

\author{
Isabel Tello Fons / Esperanza Valero Doménech ${ }^{1}$
}

Recibido: 11 de noviembre de 2016 / Aceptado: 28 de agosto de 2017

Resumen. Este análisis se propone indagar en las diferentes herramientas didácticas que los centros universitarios oficiales españoles utilizan para impartir la docencia de la traducción e interpretación a distancia. En concreto, nuestro análisis se centrará en las aplicaciones didácticas de las diferentes herramientas de los LMS (sistemas de gestión de aprendizaje) para la enseñanza de la traducción e interpretación. Herramientas de formación síncrona y asíncrona, de naturaleza audiovisual e interactiva como Moodle, Blackboard Learn and Collaborate o WebCT, entre otros, se analizarán desde el punto de vista de la didáctica de la traducción. Asimismo, se plantearán los retos a los que tanto instituciones como profesores se enfrentan en un futuro muy cercano. Nuestra intención es la de iniciar un debate sobre el futuro escenario de esta modalidad de enseñanza en nuestra área..

Palabras clave: e-learning, sistemas LMS, didáctica de la Traducción e Interpretación, Blackboard.

\section{Educational tools for the online teaching of translation and interpreting stu- dies}

\begin{abstract}
Our study will focus on studying the didactic tools the universities in Spain use to convey the teaching of online translation studies. We will carry out a comparative analysis of the systems and a description of the methodological challenges of online translation teaching.

In particular, we aim at analysing the didactic applications of different LMS -learning management systems- for translation teaching. We will go over synchronous and asynchronous, audio-visual and interactive tools such as Moodle, Blackboard Learn and Collaborate or WebCT, among others. They will be analysed from the point of view of the didactics of translation. Some day-to-day case studies in the translation classroom will be addressed as well. We finally will launch a debate on the future scenario of e-learning in Translation and Interpreting.
\end{abstract}

Keywords: e-learning, LMS systems, didactics of Translation and Interpreting, Blackboard.

Sumario: 1. Introducción: panorama de la enseñanza online del grado de traducción e interpretación. 2. Herramientas didácticas para la enseñanza online de la traducción. Diferentes sistemas LMS y breve comparación. 3. Software Blackboard Learn y Collaborate. Descripción de las herramientas para la enseñanza de la traducción. 4. Retos metodológicos. 5. Conclusiones.

Cómo citar: Tello Fons, I. y Valero Doménehc, E. (2017) Herramientas didácticas para la enseñanza universitaria de la Traducción e Interpretaciñon online, en Estudios de Traducción 7, 00.00.

$1 \quad$ Universidad Internacional de Valencia - VIU

itello@universidadviu.com / evalero@universidadviu.com 


\section{Introducción: panorama de la enseñanza online del grado de traducción e interpretación}

Los estudios superiores están atravesando un proceso de flexibilización e integración con las nuevas tecnologías que viene demandado por la sociedad. Sin embargo, autores como Salinas (2006: 1) advierten de que estos nuevos escenarios de aprendizaje exigen una fundamentación metodológica sólida. Tal como afirmaba Galán-Mañas (2009: 128), para trabajar a distancia, no se trata de virtualizar lo presencial, de ofrecerle contenidos al estudiante que tendrá que memorizar y a los que además tendrá que enfrentarse en soledad. Se trata de crear un espacio de aprendizaje virtual que conceda mayor énfasis a la actividad autónoma del estudiante. Este nuevo entorno, en principio muy ventajoso para llegar a un tipo de alumnado con un perfil algo más adulto, es algo que algunos centros de enseñanza superior han considerado y empezado a integrar en su oferta académica. Para desarrollar esta actividad formativa, los centros utilizan diferentes entornos virtuales de aprendizaje en forma de programas de software como Moodle, Blackboard, WebCT, etc.

Según el Registro de Universidades, Centros y Títulos (RUCT), de todos los grados en traducción e interpretación del estado español, sólo dos ofrecen modalidad de enseñanza online: la UOC/UVic-UCC (Universitat Central de Catalunya) y la VIU - Universidad Internacional de Valencia.

En el caso de la UOC/UVic-UCC, los alumnos que deciden cursar el grado de forma online pueden hacerlo a través del campus virtual de la UOC. Sin embargo, los alumnos que decidan estudiar el grado de forma semipresencial, cursarán algunas asignaturas de forma presencial en la UVic y otras en el campus virtual de la UOC. El resto de universidades con estudios de traducción en España, en total 29, ofrecen grados en traducción en modalidad presencial.

La UOC es una universidad pública catalana, miembro de la ACUP (Asociación Catalana de Universidades Públicas), que imparte el grado de Traducción, Interpretación y Lenguas Aplicadas en asociación con la UVic-UCC. Por su parte, la Universitat de Vic-Universitat Central de Catalunya es una universidad con un modelo propio de titularidad pública y gestión privada que imparte los estudios de traducción desde 1993 de forma presencial. En la actualidad, cuenta con el Grau en Llengües Aplicades i Traducció (Grado en Lenguas Aplicadas y Traducción) que se puede cursar de forma presencial y semipresencial gracias a la asociación con la UOC.

Por otro lado, la Universidad Internacional de Valencia-VIU es una universidad de carácter mayoritariamente privado perteneciente al Grupo Planeta que lleva operando desde el 2008 e impartiendo el Grado en Traducción e Interpretación desde el 2015.

Para llevar a cabo la formación virtual en estudios de traducción e interpretación, estas dos universidades se sirven de una serie de herramientas. La UOC/UVic-UCC utiliza una plataforma propia $a d$ hoc que está en constante evolución; la última renovación data de 2013. Los estudiantes y profesores acceden al aula a través del campus virtual de la universidad. Varias son las características destacables de esta plataforma.

En primer lugar, se compone de tres partes: la línea de tiempo (es un calendario que va destacando los eventos del curso, como las actividades que hay que entregar), la zona de la asignatura que se está cursando (donde se explican las activida- 
des que los alumnos deben realizar, las cuales se pueden descargar o imprimir) y la zona transversal de la asignatura, donde se encuentra la zona "Evaluación" (para entregar actividades o ver las notas del estudiante), "Materiales" (donde se cargan los materiales de la asignatura) y un calendario semestral. Además, existe una parte transversal donde se encuentran las redes de comunicación, como por ejemplo, el tablón de anuncios (mediante el cual se comunican estudiantes y profesores/consultores), los "foros", donde alumnos consultan dudas con otros alumnos, y los "debates", donde los alumnos discuten sobre una actividad concreta. Tanto estudiantes como profesores pueden ver qué alumnos están conectados en ese momento, acceder a sus perfiles o ver la lista de alumnos en esa asignatura. Las actividades que se han de entregar aparecen en un color diferente. Cada supuesto, problema o actividad aparece con su explicación, los recursos para poder hacerla y las soluciones. Los recursos y materiales de las asignaturas, los cuales son elaborados por un profesor o pueden ser externos y adaptados, se ofrecen en soportes diferentes, vídeo, scorm o $p d f$. Cabe destacar que el modelo de enseñanza no realiza sesiones de clase síncronas a través de su plataforma, sino que en algunas materias el profesor inserta vídeos donde él mismo imparte contenidos. Los alumnos pueden visualizarlos tantas veces como quieran puesto que son recursos que quedan en el aula.

La Universidad Internacional de Valencia dispone de la plataforma educativa Blackboard Learn y su herramienta asociada Blackboard Collaborate. Blackboard es un software de aprendizaje en línea que engloba Blackboard Learn, una plataforma de gestión de materias online, y Blackboard Collaborate, que permite realizar videoconferencias, es decir, un espacio en el que estudiantes y profesor se encuentran de manera síncrona y que sería lo más parecido a una clase tradicional. Las clases online se realizan a través de esta herramienta y el resto de la organización y contenidos de las asignaturas se gestionan a través de Blackboard Learn. Ambos están integrados, de manera que el estudiante se conecta al campus alojado en Blackboard y tiene acceso a las clases virtuales desde la misma plataforma.

De esta manera, en cualquier asignatura se imparte un número determinado de sesiones (clases) en las que el profesor imparte normalmente conceptos teóricos o descripción de actividades prácticas que los alumnos deben realizar y los alumnos escuchan, toman notas, hacen preguntas o intervienen para hacer presentaciones, corregir ejercicios, etc. como si se tratara de una clase en modalidad presencial. Ambas partes deben tener instalado el software y se necesita una webcam y un micrófono. Estas sesiones quedan grabadas para que los alumnos que no se conectan de manera síncrona sí puedan visionar la clase de forma asíncrona. Además, la herramienta Blackboard dispone de una sala virtual donde los alumnos pueden entrar y, haciendo uso de la misma herramienta de videoconferencias de Blackboard, reunirse para hacer trabajos grupales. El docente puede tener acceso a varios cursos desde una sola cuenta, puede comunicarse con los estudiantes por medio de herramientas de colaboración en línea como foros y chats, puede realizar evaluaciones y seguir el aprendizaje de sus estudiantes. Como aspecto negativo al uso de este software, Galán-Mañas destaca su alto coste, la necesidad de utilizar un navegador de Internet, y en consecuencia, de disponer de banda ancha (2009: 150).

Las plataformas de e-learning de estas dos universidades permiten la evaluación continua de las materias y el aprendizaje colaborativo. Más adelante, profundizaremos en Blackboard y describiremos cómo se utilizan las herramientas que contiene para la enseñanza de la traducción, si bien es cierto, tal como apunta González 
Davies (2010: 4) que, en la actualidad, y de manera muy concreta en la traducción y en la enseñanza de lenguas, difícilmente se puede pensar en planteamientos educativos que dejen de lado los recursos digitales disponibles.

\section{Herramientas didácticas para la enseñanza online de la traducción. Diferentes sistemas LMS y breve comparación}

Learning Management System o Sistema de Gestión de aprendizaje es el nombre que recibe un software instalado generalmente en un servidor web que se emplea para crear, administrar, almacenar y distribuir las actividades de formación virtual. Se puede utilizar como complemento de clases presenciales o para el aprendizaje a distancia. Su función principal es administrar estudiantes y dar seguimiento a su aprendizaje, participación y desempeño. Aunque existen numerosos estudios dirigidos a comparar estos sistemas (Machado y Tao 2007; Beatty y Ulasewicz 2006; Cheung 2006, entre otros) en este artículo nos vamos a centrar en describir brevemente cinco de ellos: Blackboard, Moodle, Sakai, Open EDX y Adobe Captivate Prime. De estos, Moodle, Sakai y Open EDX son el resultado de proyectos de software libre y, por lo tanto, ofrecen mayores opciones de personalización, pero requieren de un servicio de mantenimiento y desarrollo dentro de la institución para adaptarlos a las necesidades y garantizar su correcto funcionamiento. Open EDX se diferencia de Moodle y Sakai por haber sido diseñado específicamente por el Massachusetts Institute of Technology (MIT) y la Universidad de Harvard para diseñar sus cursos MOOC. La diferencia principal con respecto al resto de programas es que se trabaja a través del navegador, no es necesario instalar ningún software en los servidores de la institución. Por otro lado, Blackboard y Adobe Captivate Prime son productos comerciales que disponen de características muy atractivas para la enseñanza online. Adobe Captivate, por ejemplo, posibilita la edición de documentos colaborativamente dentro de la plataforma o las opciones de ludificación. Este concepto novedoso en los entornos digitales de educación fue definido por Deterding et al. (2011: 1) como «el uso de elementos lúdicos en contextos que no lo son». Captivate ofrece la posibilidad de que los estudiantes obtengan insignias por sus logros en el aula virtual. Por su parte, Blackboard dispone de una red social para que la comunidad de estudiantes y profesores se pueda comunicar.

Todos estos sistemas están optimizados para otros dispositivos, como tablets o teléfonos móviles, aunque las funcionalidades están limitadas con respecto a su uso en el ordenador.

Todos estos sistemas tienen buenas funciones de comunicación, tanto entre profesores y estudiantes como entre estudiantes. Algunos de ellos, como Blackboard tienen una red social en la todos los usuarios se pueden conectar. Moodle, por su parte, tienen el chat, muy útil para que dos usuarios se comuniquen de manera instantánea cuando están en la plataforma.

En cuanto al seguimiento de los estudiantes, todos estos sistemas tienen estadísticas del progreso realizado. Blackboard tiene un módulo, llamado "control del riesgo" en el que se puede ver los estudiantes que hace tiempo que no se entran en la plataforma o no han entregado las tareas y su plazo ha expirado. Adobe Captivate tiene un módulo para los estudiantes en el que pueden ver de manera gráfica su progreso y lo que les queda por terminar de cada curso. 


\section{Software blackboard learn y collaborate. Descripción de las herramientas para la enseñanza de la traducción}

A continuación se describen las herramientas que este software incluye, las cuales encontramos dentro del área correspondiente a cada asignatura, y que se utilizan para realizar la docencia de las asignaturas.

La sección llamada "Recursos y materiales" es un espacio donde los profesores cargan los recursos de las asignaturas, tanto manuales teórico/prácticos (en diferentes formatos) como vídeos docentes, artículos relacionados, enlaces a información interesante, etc. Los alumnos, no obstante, no pueden subir información en esta área. Por otro lado, la sección llamada "Actividades" es un espacio donde los alumnos pueden consultar qué actividades deben entregar, de qué tipo y en qué fecha. Aquí los alumnos cargan sus ejercicios de traducción para que el profesor pueda evaluarlos. Las actividades pueden incluir cada una rúbrica de evaluación diferente dependiendo del tipo de actividad de que se trate. Igualmente a través de este espacio los alumnos realizan los exámenes online.

En cuanto a la creación de exámenes por el profesor, éste puede elegir entre crear preguntas tipo test, tipo oración confusa, respuesta múltiple, verdadero o falso, ejercicios de rellenar huecos o de redacción. Para un examen de traducción, por ejemplo, se puede utilizar la opción de redacción. Así, el profesor introduciría el texto origen y los alumnos tendrían un espacio donde escribir el texto meta. De la misma forma, el profesor puede valorar las opciones de documentación, terminológicas o relacionadas con las técnicas de traducción utilizadas a través de preguntas que se incluirían en el mismo examen y que igualmente pueden ser de tipo respuesta abierta, test, redacción, etc. En ocasiones el profesor puede preferir que trabajen el texto en su ordenador, mediante un documento de Word y lo carguen en la plataforma en formato pdf al área de "Actividades", donde habrá una actividad creada llamada "Examen". La herramienta mediante la cual se crean los exámenes incluye la posibilidad de introducir una rúbrica o modificar una ya existente. En el caso de los estudios de traducción se puede introducir un baremo de corrección de traducciones existente o uno creado por el profesor.

Para los exámenes de lengua, la plataforma permite reproducir audios que evalúen la comprensión oral. Los estudiantes se graban a sí mismos en vídeos que cargan en la plataforma. Otra opción es que realicen un examen oral con el profesor en una sesión de videoconferencia por medio de la herramienta Blackboard Collaborate.

Una herramienta muy útil dentro de la plataforma es la herramienta «Foros», la cual incluye por defecto varias opciones: un "foro de debate", donde se discuten cuestiones planteadas por el profesor (por ejemplo, el profesor propone temas de actualidad a los que los alumnos deben reaccionar); "foro de revisión bibliográfica", donde se profundiza en textos relacionados con la parte teórica de la asignatura (si la hay); el "foro de dudas sobre los materiales docentes", el cual permite a los alumnos preguntar a otros alumnos, con la supervisión del profesor, cuestiones que no entienden sobre los materiales; el "foro de dudas sobre las actividades" permite a alumnos preguntar a otros alumnos y al profesor cuestiones que no entiende sobre las actividades que haya que realizar. Finalmente, el foro "miscelánea" se utiliza para canalizar dudas o cuestiones no incluidas en el resto de foros. El papel del profesor en los foros es activo: contesta cuando se necesita, modera los debates, lanza nuevas preguntas, propone material extra, etc. 
Puesto que la plataforma permite que se creen otros foros, el profesor podría crear un foro llamado "Puesta en común del problema X", donde los alumnos escribirían sus propuestas de traducción de un determinado problema y harían comentarios sobre el proceso. Posteriormente, el profesor valorará estas propuestas en el mismo foro o en sesión de VC. En lo que concierne a la enseñanza de la traducción especializada, los foros permiten que glosarios especializados se vayan actualizando en cualquier momento gracias a las aportaciones de los alumnos. Otros ejemplos de actividades que se pueden planificar a través de los foros son comentarios tanto macrolingüísticos como microlingüísticos sobre un texto, la puesta en común y valoración de recursos útiles para traductores (glosarios especializados, bases de datos terminológicas, herramientas informáticas, listas de distribución especializadas, sitios web de aprendizaje de idiomas), ejercicios de expresión oral en lengua extranjera, análisis sobre aspectos culturales en fragmentos audiovisuales o literarios, etc.

El software Blackboard Collaborate que permite realizar videoconferencias posibilita, como se menciona más arriba, la realización de sesiones de clase síncronas. Esta herramienta permite ver a la persona que está hablando en la parte superior izquierda y debajo de esta ventana se mostrarían las personas que están conectadas a la videoconferencia. En la parte inferior de la pantalla se sitúa un chat por el que los alumnos y el profesor se pueden comunicar a medida que se desarrolla la videoconferencia. La pizarra ocupa el mayor espacio de la pantalla, puesto que es aquí el profesor puede cargar los contenidos que haya preparado para esa clase en presentación PowerPoint, pero también enlazar a internet, a videos guardados u online o mostrar su escritorio. Las sesiones se pueden grabar para que aquellos alumnos que no se pueden conectar en el horario en el que se imparte la sesión puedan visualizarla posteriormente cuando lo deseen.

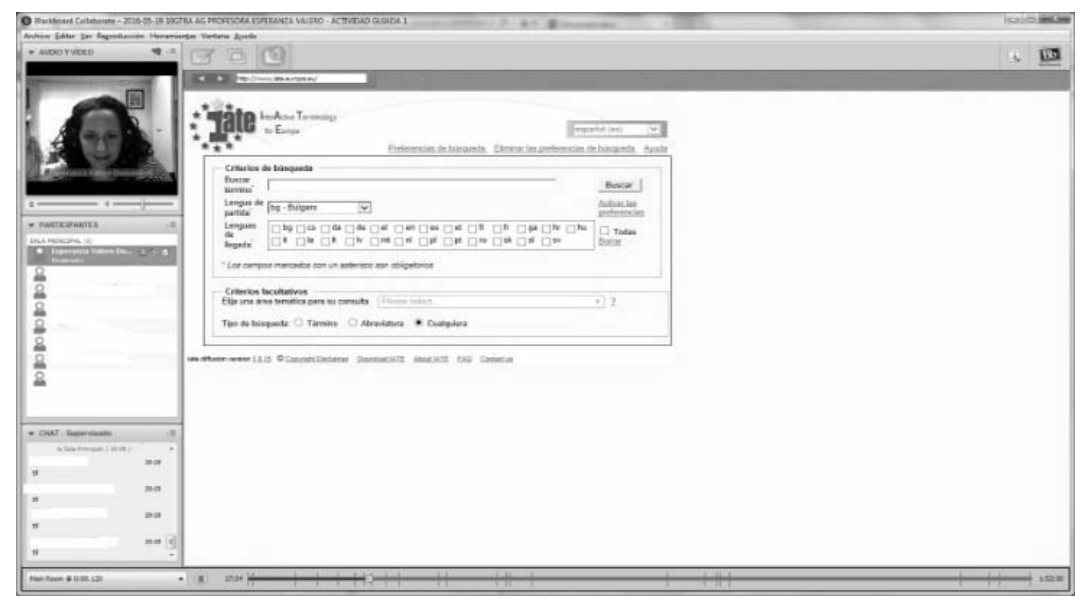

En el caso de la enseñanza de la traducción, el profesor puede utilizar la sesión para corregir una traducción con la participación del alumnado. De esta manera, los alumnos (los cuales pueden intervenir en la videoconferencia usando sus webcams y micrófonos) leerían sus versiones y comentarían las soluciones delante de sus compañeros. El resto de alumnos también puede reaccionar a estas intervenciones a través del chat, de forma que el resto de la clase y el profesor lo lean, o igualmente 
pueden tomar la palabra cuando la intervención de su compañero haya terminado. En la pizarra, el profesor puede ir escribiendo la versión consensuada que se va creando a partir de las aportaciones de los alumnos.

Las salas internas son opciones que encontramos dentro de la herramienta de videoconferencias. El profesor puede encomendar a los alumnos la realización de una actividad grupal para la cual se necesite que los alumnos trabajen en grupos en un espacio diferente al de la sala principal. De esta manera, la opción "Crear salas internas" permite que el profesor cree tantas salas como se necesiten y los alumnos pueden moverse a las distintas salas con sus compañeros de grupo. El profesor establecerá un tiempo en el que los alumnos irán trabajando en grupos y después de este tiempo serán llevados a la sala principal. El profesor podrá moverse virtualmente de sala en sala para interesarse por la marcha del trabajo o responder dudas. Cuando el profesor esté en la sala principal, los alumnos de las salas podrán llamarle para que acuda a la sala privada.

Una actividad que puede resultar interesante es la "crítica de traducciones". El profesor, después de pedir el envío de la traducción de un texto a sus alumnos, puede crear salas internas y en cada una de las pizarras de las salas cargar el texto traducido de un alumno diferente. Después de dejar un tiempo para que, con un baremo de corrección colgado en la pizarra de la sala principal o proporcionado a los alumnos de antemano, estos corrigen y clasifican los errores de las traducciones de sus compañeros. Pasado este tiempo, el profesor envía a todos los alumnos a la sala principal y se pondrían en común los errores más recurrentes.

Por último, las videoconferencias permiten realizar tutorías individuales en las que resolver dudas específicas o revisar exámenes.

\section{Retos metodológicos}

La formación continua que se exige actualmente en el mundo laboral hace que esta modalidad de aprendizaje sea idónea para compaginar la formación con el trabajo. El modelo pedagógico es muy flexible puesto que se adapta a las necesidades de cada estudiante, sitúa al alumno en el centro de la actividad formativa y por tanto resulta óptimo tanto para un perfil de alumno adulto como para uno más joven que no resida cerca de un centro donde se impartan los estudios de traducción.

Uno de los objetivos del Espacio Europeo de Educación Superior (EEES) es lograr que los estudiantes progresen profesionalmente, y en este sentido, el e-learning responde a las necesidades reales de las empresas en un mercado globalizado, puesto que la enseñanza online potencia el aprendizaje y uso de las TICS. Sin duda, el manejo de la tecnología de forma continua va a ser beneficioso de cara a la búsqueda de un trabajo posterior. A partir de aquí, la enseñanza de los estudios de traducción e interpretación a través de la modalidad online se enfrenta a una serie de retos metodológicos como pueden ser las cuestiones formales propias de los sistemas de gestión de aprendizaje, por ejemplo, la claridad de los software, los cuales deben ser cada vez más intuitivos para potenciar la eficiencia de la enseñanza. Asimismo, se hace necesaria la inclusión de herramientas online que beneficien el aprendizaje colaborativo: de edición de textos online (Google Docs o Microsoft OneDrive), de comunicación instantánea (Skype, Google Hangouts, el que permite videollamadas grupales, o aplicaciones móviles como Whatsapp), que posibiliten la 
comunicación inmediata de los estudiantes fuera de las sesiones de videoconferencia, por ejemplo, para realizar traducciones en grupo o proyectos de traducción de mayor envergadura. Igualmente sería muy importante que todo lo anterior estuviera integrado en la plataforma o en el campus para mayor comodidad de los usuarios.

Además de lo anterior, la enseñanza online debe utilizar programas de software que tengan la capacidad de mejora continua para ir adquiriendo las nuevas herramientas tecnológicas que el mercado ofrezca, luego la revisión constante del software y de sus herramientas se hace necesaria. Por último, otro de los retos a los que se enfrenta este tipo de formación, y algo en lo que ya va encaminado, es a la consolidación de un rol de profesor como guía de los alumnos y no como fuente de todo conocimiento y persona a la que consultar para todo. Los foros, supervisados por el profesor, facilitan esta tarea. El profesor, ahora gestor de recursos, deberá modificar su preparación profesional para adecuarse a este nuevo contexto. Igualmente será necesario un cambio en el rol del alumno, el cual deberá ser capaz de seleccionar y organizar la información para ir formándose en esta sociedad de la información.

Centrándonos en nuestra área, existen retos metodológicos específicos a los que nos tenemos que enfrentar en las diferentes asignaturas, desde la enseñanza de lenguas online hasta la interpretación.

En cuanto a la enseñanza de lenguas, al no existir un método de lenguas tradicional en papel, será importante que las aulas incluyan acceso a materiales digitales que hoy en día ofertan las diferentes editoriales. Esta necesidad enlaza con la enseñanza de lenguas asistida por ordenador (de sus siglas en inglés CALL), la cual nació en los años 60 del pasado siglo y puede dividirse en tres etapas: la etapa estructural, la etapa comunicativa y la etapa integrativa. Basándose en estos enfoques, los productos CALL han ido diseñando sus herramientas. En la primera etapa el alumno practicaba ejercicios basados en la repetición y el ordenador era una especie de tutor electrónico que nunca juzgaba y que dejaba que los alumnos aprendieran cada uno a su ritmo (Warschauer y Healey 1998: 57). En la etapa actual los productos CALL permiten a los usuarios aprender con materiales reales y satisfacer los gustos de un alumnado que es actualmente usuario «nativo» de la tecnología (Warschauer 2004: 4). Tal como indica Warschauer: «the purpose of studying English thus becomes not just to acquire it as an internal system, but to be able to use English to have a real impact on the world» (2000: 12). Sin duda, los programas multimedia que incluyen herramientas de reconocimiento de voz pueden sumergir a los usuarios en un ambiente rico para la práctica de la lengua (Yang 2010: 902).

Algunos autores afirman que la necesidad en la actualidad de implementar la tecnología sin tener en cuenta otros aspectos cruciales en la enseñanza como es el apoyo pedagógico que aporta un profesor (Bax 2003: 25) puede llegar a provocar una reacción negativa en escuelas, alumnos y profesores. Tal como exponen Coryell y Clup (2007) existen páginas web que funcionan como redes sociales para aprender idiomas o software específico para la adquisición de segundas lenguas. Sobre el papel de internet en la enseñanza de lenguas, Ruiz (2005: 65) afirma que los cibergéneros han iniciado una generación discursiva que puede ser utilizada con fines pedagógicos en CALL y TICs. Estos materiales basados en tecnologías incluyen ejercicios para practicar las diferentes destrezas lingüísticas y su realización puede ser supervisada por el profesor para hacer el seguimiento del alumno. Los ejercicios pueden ir desde archivos en pdf enriquecidos hasta materiales más interactivos.

La enseñanza y aprendizaje online de la interpretación presenta retos diferentes 
a los que se observan en la enseñanza presencial, puesto que los estudiantes ya no se encuentran en las cabinas de un laboratorio de interpretación, sino en una videoconferencia. Tal como exponen en su trabajo Ko y Chen (2011: 139) la principal dificultad es la falta de interacción visual entre profesores y alumnos. Sin embargo, con herramientas actuales para clases virtuales se puede paliar el efecto que esta carencia tiene en la enseñanza. En este caso, el profesor puede enviar a cada estudiante a una sala individual y reproducir el audio en todas las salas, el estudiante va interpretando y el profesor pasa por las salas para observar y dar feedback al estudiante. Otra opción que pueden barajar los profesores es la de planificar sesiones breves para grupos reducidos y proporcionar una docencia más individualizada.

Otra área que requiere especial planificación en estos estudios es la de las tecnologías. Los estudiantes no utilizan los ordenadores del laboratorio, sino que trabajan en sus propios equipos donde se han instalado las licencias de los programas adquiridos. De esto se derivan ciertas dificultades como la configuración de cada equipo, problemas de interacción con otros programas instalados, incapacidad del profesor de ver exactamente qué ocurre en el ordenador del estudiante, etc. Sin embargo, cabe destacar la ventaja de que los estudiantes tengan el software en su ordenador y lo puedan utilizar en cualquier momento, sin depender del horario del laboratorio. Otra opción utilizada por numerosos centros de enseñanza online son las plataformas de traducción colaborativas. Constituyen herramientas TAO en la nube que, sin necesidad de instalarse ningún programa, dan acceso a memorias de traducción y otros recursos que se puedan utilizar simultáneamente por numerosos usuarios. Como concluye Monti (2012) incluir estas herramientas en el aula permite a los estudiantes desarrollar nuevas habilidades profesionales de teletrabajo y también de traducción paralela y post-edición.

\section{Conclusiones}

Es innegable que la docencia online está avanzando terreno en los estudios superiores. Si pensamos en las propuestas de la Declaración de Bolonia y en la implantación del Espacio Europeo de Educación Superior, nos damos cuenta de que el modelo educativo centrado en el trabajo del estudiante y no tanto en el concepto de horas de clase refuerza aún más este planteamiento (González-Davies 2010: 6). Las ventajas que ofrece son evidentes, sin embargo, no está carente de dificultades que afectan a varios niveles, desde cuestiones generales pedagógicas a dificultades específicas de una materia en particular pasando por el descuido que aún impera en las políticas institucionales, las cuales reducen el desarrollo de competencias al uso de aplicaciones y herramientas, pero no asocian esto a referentes teóricos y pedagogías que justifiquen su uso. De ahí que Ricaurte-Quijano y Carli-Álvarez (2016: 64) argumenten que el desarrollo de competencias digitales deba concebirse de manera integral y asociarse con un conjunto de principios relativos a la producción del conocimiento y la cooperación en la era digital.

El primer paso al que se debe enfrentar una institución es seleccionar el software LMS más adecuado u optar por desarrollar un sistema propio, lo que deriva en decisiones tan importantes como el presupuesto disponible, la adquisición de soporte técnico online o el diseño de un protocolo de comunicación con los estudiantes, entre otros, todos ellos aspectos no contemplados en la enseñanza de la traducción 
convencional (Ko 2012: 24). Posteriormente, es necesario adoptar una serie de decisiones que afectarán a la metodología, como por ejemplo en lo que se refiere a la selección de los módulos que se van a utilizar y los materiales que se van a crear para el estudiantado. Una vez iniciado el proceso, la continua revisión y evolución de metodologías y herramientas es necesaria para poder resolver las dificultades de esta nueva modalidad de docencia en nuestra área.

\section{Referencias bibliográficas}

Adobe Captivate Prime [software]. (2016). [Retrieved from: http://www.adobe.com/es/products/captivateprime.asdf]

Beatty, B., \& Ulasewicz, C., «Faculty perspectives on moving from Blackboard to the Moodle learning management system». TechTrends, 50(4), (2006), 36-45.

Blackboard (12.6.6.7847) [software]. (2000-2015). [Retrieved from: http://www.blackboard.com/]

Cheung, K. S., «A Comparison of WebCT, Blackboard and Moodle for the teaching and learning of continuing education courses». Enhancing learning through technology, (2006), 219-228.

Coryell, J. E., \& Chlup, D. T. «Implementing e-learning components with adult English language learners: Vital factors and lessons learned», Computer Assisted Language Learning, 20(3), (2007), 263-278.

Deterding, S., Khaled, R., Nacke, L.E., Dixon, D. (2011). «Gamification: Toward a Definition». In CHI 2011 Gamification Workshop Proceedings, Vancouver, BC, Canada 2011.

Galán-Mañas, A., La enseñanza de la traducción en la modalidad semipresencial. Tesis Doctorales en Xarxa (TDX). Barcelona: Universitat Auònoma de Barcelona, (2009). [Retrieved from: http://hdl.handle.net/10803/5275].

Cánovas, M., González Davies, M. y Keim, L., Acortar distancias. Las TIC en la clase de traducción y de lenguas extranjeras. Barcelona: Ediciones Octaedro 2010.

Ko, L. «Teaching Translation Online: A Reflective Study», Translation Quarterly, 63 (2012), 1-26.

Ko, L. y Chen, N., «Online-interpreting in synchronous cyber classrooms», Babel, 57(2), (2011), 123-143.

Machado, M., \& Tao, E., «Blackboard vs. Moodle: Comparing user experience of learning management systems». In 37th Annual Frontiers in Education Conference-Global Engineering: Knowledge Without Borders, Opportunities Without Passports, Milwaukee, West Indies 2007.

Moodle (3.1) [software]. (2016). [Retrieved from: http://www.moodle.org]

Monti, J. «Translators' knowledge in the Cloud: The New Translation Technologies». In International Symposium on Language and Communication: Research Trends and Challenges (ISLC), İzmir University, Turkey 2012.

Open EDX (0.7) [software]. (2016). [Retrieved from: http://open.edx.org/]

Registro de Universidades, Centros y Títulos (RUCT). Sección de títulos [Retrieved from: https://www.educacion.gob.es/ruct/consultaestudios?actual=estudios]

Ricaurte-Quijano, P. y Carli-Álvarez, A., «El proyecto Wiki Learning: Wikipedia como entorno de aprendizaje abierto», Comunicar. Revista científica de educación, 49 - XXIV, (2016), 61-69. 
Ruiz Madrid, $\mathrm{M}^{\mathrm{a}}$ N. Learner Autonomy in Computer Assisted Language Learning. A Comparative Case Study of Learners'Behaviour in the English as a Foreign Language Context. Castellón de la Plana: Universitat Jaume I, (2005). [Retrieved from: http://hdl.handle.net/10234/29578].

Sakai (0.7) [software]. (a2016). [Retrieved from: https://sakaiproject.org/]

Salinas, J., "Innovación docente y uso de las TIC en la enseñanza universitaria», Revista Universidad y Sociedad del Conocimiento, 1-1, (2004), 1-16.

Warschauer, M. and Healey, D., «Computers and language learning: an overview». Language Teaching, 31 (1998), 57-71.

Warschauer, M. «CALL for the 21 st Century», In IATEFL and ESADE Conference, Barcelona 2000.

Warschauer, M. «Technological change and the future of CALL», en: Fotos, S y Brown, C (eds.), New Perspectives on CALL for Second and Foreign Language Classrooms, Mahwah, NJ: Lawrence Erlbaum Associates 2004, 15-25.

Yang, Y., «Computer-assisted Foreign Language Teaching: Theory and Practice», Journal of Language Teaching and Research, 1-6, (2010), 909-912. 